\title{
THE BOUNDED AND THIN WHITEHEAD THEOREMS
}

\author{
DOUGLAS R. ANDERSON AND HANS JØRGEN MUNKHOLM
}

(Communicated by James E. West)

\begin{abstract}
This paper deals with finite-dimensional CW complexes equipped with reference maps to a fixed metric space and maps between such complexes that respect the reference maps up to a bounded distortion. We prove two Whitehead Theorems for such maps $f$. The Bounded Whitehead Theorem allows one to decide whether $f$ is a bounded homotopy equivalence. The Thin Whitehead Theorem allows one to decide when a map of bound zero admits homotopy inverses of arbitrarily small bound (also on the homotopies). Both theorems come in two versions: One that deals with homotopy in all dimensions; one where homotopy in dimensions at least two is replaced by homology of "universal covers".
\end{abstract}

\section{INTRODUCTION}

Let $Z$ be a metric space with metric $\rho$. For any $z \in Z$ and any $r \geq 0$, let $B(z, r)=\{y \in Z \mid \rho(y, z) \leq r\}$. For simplicity, we denote $B(z, r)$ by $B$ and for any number $d \geq 0$, let $B^{d}=B(z, r+d) .1$

A pair $(X, p)$ with $X$ a finite-dimensional $\mathrm{CW}$ complex and $p: X \rightarrow Z$ a continuous map is called a bounded $C W$ complex over $Z$ if there is a number $d_{X}$ with $\operatorname{diam} p(e) \leq d_{X}$ for every cell $e$ in $X$. A map $f:(X, p) \rightarrow(Y, q)$ between two such spaces is bounded with bound $d(f) \geq 0$ if $\rho(q f(x), p(x)) \leq$ $d(f)$ for any $x \in X$ (cf. [Pe] or [AM1, p. 44]). It is called a homotopy equivalence of bound $d$ if $d(f) \leq d$ and there are bounded maps $g:(Y, q) \rightarrow$ $(X, p), H:(X \times I, p \pi) \rightarrow(X, p)$, and $G:(Y \times I, q \pi) \rightarrow(Y, q)$, all of bound $d$, with $H: 1_{X} \simeq g f$ and $G: 1_{Y} \simeq f g$. Here $\pi$ is a projection on the first factor.

Let $f:(X, p) \rightarrow(Y, q)$ be a bounded map. We say that $\pi_{n}(f)$ is an $(a, b)$ isomorphism if $a$ is a bound for $f$ and for every ball $B \subseteq Z$ and every

Received by the editors March 2, 1990 and, in revised form, June 10, 1991.

1980 Mathematics Subject Classification (1985 Revision). Primary 55P10.

The first author was partially supported by the NSF under grant number DMS-8803149.

The second author was partially supported by SNF (Denmark) under grant number 11-7792.

${ }^{1}$ Note that one may have $B(z, r)=B(w, s)$, but $B(z, r+d) \neq B(w, s+d)$, so $B^{d}$ really may depend on $(z, r)$, and not just on the set $B=B(z, r)$. 
$x \in p^{-1} B, \operatorname{im} j_{1 *} \subseteq \operatorname{im} f_{1 *}$ and $\operatorname{ker} f_{0 *} \subseteq \operatorname{ker} j_{0 *}$ in the diagram

$$
\begin{array}{cc}
\pi_{n}\left(p^{-1} B, x\right) \stackrel{f_{0 *}}{\longrightarrow} & \pi_{n}\left(q^{-1} B^{a}, f(x)\right) \\
j_{0 *} \downarrow & \downarrow_{1 *}^{j_{1 *}} \\
\pi_{n}\left(p^{-1} B^{b}, x\right) \stackrel{f_{1 *}}{\longrightarrow} \pi_{n}\left(q^{-1} B^{a+b}, f(x)\right)
\end{array}
$$

If $n=-1$, we say that $\pi_{-1}(f)$ is an $(a, b)$-isomorphism if $a$ is a bound for $f$ and for every ball $B \subseteq Z$ with $q^{-1} B \neq \varnothing, p^{-1} B^{b} \neq \varnothing$.

We say that $H_{n}(\tilde{f})$ is an $(a, b)$-isomorphism if $a$ is a bound for $f$, and for every ball $B \subseteq Z$ and every $x \in p^{-1} B, \operatorname{im} \tilde{j}_{1 *} \subseteq \operatorname{im} \tilde{f}_{1 *}$ and $\operatorname{ker} \tilde{f}_{0 *} \subseteq \operatorname{ker} \tilde{j}_{0 *}$ in the diagram

$$
\begin{array}{cc}
H_{n}(\tilde{X}(x, B)) \stackrel{\tilde{f}_{0 *}}{\longrightarrow} & H_{n}\left(\tilde{Y}\left(f(x), B^{a}\right)\right) \\
\tilde{j}_{0 *} \downarrow & \downarrow_{j_{1 *}} \\
H_{n}\left(\tilde{X}\left(x, B^{b}\right)\right) \stackrel{\tilde{f}_{1 *}}{\longrightarrow} & H_{n}\left(\tilde{Y}\left(f(x), B^{a+b}\right)\right)
\end{array}
$$

Here $\tilde{X}(x, B)=P\left(p^{-1} B, x\right) / \simeq$ is the space of paths in $p^{-1} B$ starting at $x$ modulo homotopy relative to endpoints, the other spaces are defined similarly, and $\tilde{f}_{i}$ and $\tilde{j}_{i}(i=0,1)$ are induced by the obvious maps of path spaces. We think of $\tilde{X}(x, B)$ as the "universal cover" of the component of $p^{-1} B$ containing $x$ (cf. [AM2, Example 1.12]).

This paper proves the following two theorems:

Bounded Whitehead Theorem. A bounded map $f:(X, p) \rightarrow(Y, q)$ between bounded $C W$ complexes over a metric space $Z$ is a bounded homotopy equivalence if and only if there is a pair $(a, b)$ so that $\pi_{n}(f)$ is an $(a, b)$-isomorphism for $n=-1,0,1$ and either

(1) $\pi_{n}(f)$ is an $(a, b)$-isomorphism for all $n \geq 2$; or

(2) $H_{n}(\tilde{f})$ is an $(a, b)$-isomorphism for all $n \geq 2$.

Furthermore for every pair of nonnegative integers $(N, M)$ there is a linear function $L_{N, M}\left(d_{1}, d_{2}, d_{3}, d_{4}\right)$ such that if $f$ satisfies the above conditions relative to $(a, b)$ then $f$ is a bounded homotopy equivalence with bound $L_{N, M}\left(a, b, d_{X}, d_{Y}\right)$, where $N=\operatorname{dim} X, M=\operatorname{dim} Y$, and $d_{X}$ and $d_{Y}$ are bounds on the cell sizes in $X$ and $Y$, respectively.

A bounded map $f:(X, p) \rightarrow(Y, q)$ is called a thin homotopy equivalence if $d(f)=0$ and $f$ is a bounded homotopy equivalence of bound $\varepsilon$ for every $\varepsilon>0$.

Thin Whitehead Theorem. A bounded map $f:(X, p) \rightarrow(Y, q)$ between bounded $C W$ complexes over a metric space $Z$ is a thin homotopy equivalence 
if and only if for every $\varepsilon>0, \pi_{n}(f)$ is a $(0, \varepsilon)$-isomorphism for $n=-1,0,1$ and either

(1) $\pi_{n}(f)$ is a $(0, \varepsilon)$-isomorphism for all $n \geq 2$; or

(2) $H_{n}(\tilde{f})$ is a $(0, \varepsilon)$-isomorphism for all $n \geq 2$.

\section{REDUCTION TO THE CASE OF AN INCLUSION}

In this section we use a mapping cylinder argument to reduce the proof of the "if" part of the Bounded Whitehead Theorem to the case where $f:(X, p) \rightarrow$ $(Y, q)$ is the inclusion of a subcomplex and $p=q f$. To make the relevant mapping cylinder into a bounded $\mathrm{CW}$ complex, we prove a Bounded Cellular Approximation Theorem. We also study the behavior of the hypotheses and conclusion of the Bounded Whitehead Theorem under the elementary changes needed to pass from $f$, via a cellular approximation to $f$, to an inclusion. The first two lemmas address the latter question. Although the bounds obtained are not necessarily best possible, the important feature is that they depend linearly on the given bounds.

Lemma 1.1. Let $f, f^{\prime}:(X, p) \rightarrow(Y, q)$ and $g:(Y, q) \rightarrow(W, r)$ be bounded maps and $H: f \simeq f^{\prime}$ be a bounded homotopy of bound $c$.

(i) If $f$ and $g$ are homotopy equivalences of bounds $a$ and $b$ respectively, then $g f$ is a homotopy equivalence of bound $2(a+b)$.

(ii) If $f$ is a homotopy equivalence of bound $a$ and $c \geq a$, then $f^{\prime}$ is $a$ homotopy equivalence of bound $a+c$.

(iii) If $a$ is a bound for $f$, then $1_{X}:(X, p) \rightarrow(X, q f)$ and $1_{X}:(X, q f) \rightarrow$ $(X, p)$ both have bound $a$. Hence they are homotopy equivalences (indeed, homeomorphisms) of bound $a$.

Proof. We leave the details to the reader after noticing that if $H: f \simeq f^{\prime}$ and $K: f^{\prime} \simeq f^{\prime \prime}$, then the combined homotopy $H * K: f \simeq f^{\prime \prime}$ has bound $d(H * K)=$ $\max (d(H), d(K))$.

Lemma 1.2. Let $f, f^{\prime}, g$, and $H$ be as in Lemma 1.1.

(i) Suppose $\pi_{n}(f)$ is an $(a, b)$-isomorphism. If $a^{\prime} \geq a$, then $\pi_{n}(f)$ is an $\left(a^{\prime}, b+a^{\prime}-a\right)$-isomorphism. If $a^{\prime \prime} \leq a$ and $a^{\prime \prime}$ is $a$ bound for $f$, then $\pi_{n}(f)$ is an $\left(a^{\prime \prime}, b+a-a^{\prime \prime}\right)$-isomorphism. If $b^{\prime} \geq b$, then $\pi_{n}(f)$ is an $\left(a, b^{\prime}\right)$ isomorphism. If $\pi_{n}(g)$ is a $(d, e)$-isomorphism, then $g f$ is an $(a+d, b+e)$ isomorphism.

(ii) If $f$ is a homotopy equivalence of bound $a$, then $\pi_{n}(f)$ is an $(a, 4 a)$ isomorphism.

(iii) If $\pi_{n}(f)$ is an $(a, b)$-isomorphism, then $\pi_{n}(f:(X, q f) \rightarrow(Y, q))$ is a $(0,2 a+b)$-isomorphism.

(iv) If $\pi_{n}(f)$ is an $(a, b)$-isomorphism and $c \geq a$, then $\pi_{n}\left(f^{\prime}\right)$ is $a(c, b+$ $c-a)$-isomorphism.

Similar results hold for $H_{n}\left({ }^{\sim}\right)$. 
Proof. We prove only the "surjectivity" part of (ii). Let $h:(Y, q) \rightarrow(X, p)$ be a homotopy inverse for $f$ and $H: 1_{X} \simeq h f$ and $K: 1_{Y} \simeq f h$ be homotopies of bound $a$. For any $x \in X$ and $y \in Y$, let $\chi(x)=H(x,-)$ and $\kappa(y)=$ $K(y,-)$. Then $\chi(x)$ is a path in $X$ from $x$ to $h f(x)$ and $\kappa(y)$ is a path in $Y$ from $y$ to $f h(y)$. Let $B \subseteq Z$ be a ball and $x \in p^{-1} B$. Then $\gamma=\gamma(x)=$ $f(\chi(x))^{-1} * \kappa(f(x))$ is a loop in $q^{-1} B^{3 a}$ based at $f(x)$ and for any $r \geq a$, there is a commutative diagram

$$
\begin{array}{ccc}
\pi_{n}\left(p^{-1} B^{a+r}, h f(x)\right) \stackrel{h_{*}}{ } & \pi_{n}\left(q^{-1} B^{r}, f(x)\right) \\
\chi(x)_{*}{ }^{-1} \downarrow & \left\lfloor\gamma_{*} j_{*}\right. \\
\pi_{n}\left(p^{-1} B^{a+r}, x\right) & \longrightarrow \pi_{n}\left(q^{-1} B^{2 a+r}, f(x)\right)
\end{array}
$$

where $j$ is an inclusion. Thus, for any $\alpha \in \pi_{n}\left(q^{-1} B^{r}, f(x)\right), \gamma_{\#} j_{*}(\alpha) \in \operatorname{im} f_{*}$. If $\beta \in \pi_{n}\left(q^{-1} B^{a}, f(x)\right)$, let $\alpha=\gamma_{\#}^{-1} j_{*}(\beta) \in \pi_{n}\left(q^{-1} B^{3 a}, f(x)\right)$. Then for $r=3 a, j_{*}(\beta)=\gamma_{\# j_{*}}(\alpha) \in \operatorname{im}\left[f_{*}: \pi_{n}\left(p^{-1} B^{4 a}, x\right) \rightarrow \pi_{n}\left(q^{-1} B^{5 a}, f(x)\right)\right]$ by the above. The "surjectivity" of $\pi_{n}(f)$ follows.

A pair of bounded $C W$ complexes over $Z$ is a triple $(Y, C, q)$ with $(Y, q)$ a bounded CW complex and $C$ a subcomplex. We say $\pi_{n}(Y, C, q) b$-vanishes if for every ball $B \subseteq Z$ and every $c \in q^{-1} B \cap C, \pi_{n}\left(q^{-1} B, q^{-1} B \cap C, c\right)$ $\rightarrow \pi_{n}\left(q^{-1} B^{b}, q^{-1} B^{b} \cap C, c\right)$ is trivial. We extend this terminology to the case $n=-1$ by saying that $\pi_{-1}(Y, C, q)$ b-vanishes if $\pi_{-1}(i)$ is a $(0, b)$ isomorphism where $i:(C, q \mid C) \rightarrow(Y, q)$ is the inclusion. There are similar notions for bounded $\mathrm{CW}$ complexes, rather than pairs, and for homology.

The following version of Lemma 10.6 [AM1, p. 93] is used in proving the Bounded Cellular Approximation Theorem. We consider pairs of bounded CW complexes $(X, A, p)$ and $(Y, C, q)$, and for any integer $n \geq 0$, set $X^{[n]}=$ $A \cup X^{(n)}$ where $X^{(n)}$ is the $n$-skeleton of $X$.

Lemma 1.3. Let $h:\left(X^{[n]} \times 0 \cup X^{[n-1]} \times I, X^{[n-1]} \times 1, p \pi\right) \rightarrow(Y, C, q)$ be a map of bound $d(h)$. Suppose that $\pi_{n}(Y, C, q) d$-vanishes for some $d \geq 0$, and if $n=0$, suppose also that $\pi_{-1}(Y, C, q) d$-vanishes. Then there is an extension of $h, H:\left(X^{[n]} \times I, X^{[n]} \times 1, p \pi\right) \rightarrow(Y, C, q)$, of bound $d(H)=3 d(h)+2 d_{X}+3 d$. Proof. It suffices to extend $h$ over $e \times I$ for any $n$-cell $e$ of $X-A$. Let $\phi:\left(D^{n}, S^{n-1}\right) \rightarrow\left(X^{[n]}, X^{[n-1]}\right)$ be a characteristic map for $e$, and let $\psi$ be the composite

$$
\begin{aligned}
& \left(D^{n} \cup S^{n-1} \times I, S^{n-1} \times 1\right) \\
& \quad \stackrel{\phi \cup\left(\phi \mid S^{n-1}\right) \times 1}{\longrightarrow}\left(X^{[n]} \times 0 \cup X^{[n-1]} \times I, X^{[n-1]} \times 1\right) \stackrel{h}{\rightarrow}(Y, C) .
\end{aligned}
$$

If $n \geq 1$, we let $c \in C$ be $\psi(*, 1)$ where $* \in S^{n-1}$ is the basepoint. If $n=0$, we use the $d$-vanishing of $\pi_{-1}(Y, C, q)$ to choose a point $c \in C$ with $\rho\left(q(c), q \psi\left(D^{0}\right)\right) \leq d$. In either case, let $z=q(c) \in Z$ and let

$$
B=B\left(z, 2 d(h)+d_{X}+d\right) .
$$

Then $\psi$ represents an element $[\psi] \in \pi_{n}\left(q^{-1} B, q^{-1} B \cap C, c\right)$. Since this group (or pointed set) $d$-vanishes, [ $\psi]$ maps to zero when we replace $B$ by $B^{d}$; so 
$h \mid(e \times 0 \cup \partial e \times I)$ extends to a map $e \times I \rightarrow q^{-1} B^{d}$. This defines $H$ with the bound claimed.

Using Lemma 1.3 inductively, one easily proves the following version of Theorem 10.1 [AM1, p. 92]. Cells of dimension $>r$ can be handled because $\pi_{n}(Y, Y, q)$ 0-vanishes.

Proposition 1.4. For any integer $N \geq 0$ there is a linear function $L_{N}\left(d_{1}, d_{2}, d_{3}\right)$ for which the following holds: Let $f:(X, A, p) \rightarrow(Y, C, q)$ be a map of bound $d(f)$ between pairs of bounded $C W$ complexes with $\operatorname{dim}(X-A)=N$. Suppose also that for some integer $r \geq 0, \pi_{n}(Y, C, q) d$-vanishes for $-1 \leq n \leq r$. Then there exists a homotopy $H:((X, A) \times I, p \pi) \rightarrow(Y, C, q)$ relative to $A$ and with bound $d(H)=L_{N}\left(d(f), d_{X}, d\right)$ such that $H(-, 0)=f$ and $g=H(-, 1)$ maps $X^{[r]}$ into $C$.

Applying Proposition 1.4 to the identity map of $(Y, C, q)$ yields the first of the following corollaries.

Corollary 1.5. For any integer $N \geq 0$ there is a linear function $L_{N}\left(d_{1}, d_{2}\right)$ for which the following holds: Let $(Y, C, q)$ be a pair of bounded $C W$ complexes with $\operatorname{dim}(Y-C)=N$ and for which $\pi_{n}(Y, C, q)$ d-vanishes for $-1 \leq n \leq N$. Then there is a strong deformation retraction of $(Y, q)$ onto $(C, q \mid C)$ of bound $L_{N}\left(d_{Y}, d\right)$

Corollary 1.6 (Bounded Cellular Approximation Theorem). For any pair $(N, M)$ of nonnegative integers, there is a linear function $L_{N, M}\left(d_{1}, d_{2}, d_{3}\right)$ for which the following holds: Let $f:(X, A, p) \rightarrow(Y, C, q)$ be a map of bound $d(f)$ between pairs of bounded $C W$ complexes with $\operatorname{dim}(X-A)=N, \operatorname{dim}(Y-C)$ $=M$. Then there exists a homotopy $H:((X, A) \times I, p \pi) \rightarrow(Y, C, q)$, relative to $A$ and of bound $d(H)=L_{N, M}\left(d(f), d_{X}, d_{Y}\right)$, such that $H(-, 0)=f$, and $g=H(-, 1)$ maps $X^{[n]}$ into $Y^{[n]}$ for every $n \geq 0$.

Proof. For each ball $B \subseteq Z$, let $Y_{B}$ be the smallest subcomplex of $Y$ containing $q^{-1} B$. Then $q^{-1} B \subseteq Y_{B} \subseteq q^{-1} B^{M d_{Y}}$, so $\pi_{n}\left(Y, Y^{[r]}, q\right) \quad M d_{Y}$-vanishes for $-1 \leq n \leq r$ and $r \geq 0$ (an easy special argument is needed for $n=-1$ ).

We assume, for some $r \geq-1$, that we have a homotopy $H_{r}:((X, A) \times$ $I, p \pi) \rightarrow(Y, C, q)$ relative to $A$ and of bound $d\left(H_{r}\right)$ and such that $H_{r}(-, 0)$ $=f$ and $g_{r}=H_{r}(-, 1)$ maps $X^{[n]}$ into $Y^{[n]}$ for $-1 \leq n \leq r$. We apply Proposition 1.4 to $g_{r}:\left(X, X^{[r]}, p\right) \rightarrow\left(Y, Y^{[r+1]}, q\right)$ and get a homotopy $h_{r}: g_{r} \simeq g_{r+1}$ relative to $X^{[r]}$, of bound $d\left(h_{r}\right)=L_{N}\left(d\left(g_{r}\right), d_{X}, M d_{Y}\right)$ and such that $g_{r+1}\left(X^{[r+1]}\right) \subseteq Y^{[r+1]}$. Let $H_{r+1}$ be $H_{r}$ followed by $h_{r}$. Since $H_{-1}=f \pi$, $d\left(H_{-1}\right)=d(f)$. Since $d\left(g_{r}\right)=d\left(H_{r}\right)$, an easy induction establishes the desired type of bound for $H=H_{N}$.

Let $f:(X, p) \rightarrow(Y, q)$ satisfy the conditions in the ("if" part of the) Bounded Whitehead Theorem for the pair $(a, b)$. By Corollary 1.6, there is a cellular map $g$ homotopic to $f$ via a homotopy of bound $L_{N, M}\left(a, d_{X}, d_{Y}\right)$. By Lemma 1.1 (ii), it suffices to obtain the conclusion for $g$. By Lemma 1.2 (iv), if $\pi_{n}(f)$ is an $(a, b)$-isomorphism, then $\pi_{n}(g)$ is an $(L, b+L-a)$ isomorphism for $L=L_{N, M}\left(a, d_{X}, d_{Y}\right)$. A similar statement holds for $H_{n}(\tilde{f})$ and $H_{n}(\tilde{g})$.

Hence we may assume that $f$ is cellular so that its mapping cylinder has a 
natural CW structure. The standard factorization of $f$ is

$$
(X, p) \stackrel{1_{X}}{\longrightarrow}(X, q f) \stackrel{i}{\longrightarrow}(M(f), q r) \stackrel{r}{\longrightarrow}(Y, q)
$$

where $1_{X}$ is a homotopy equivalence of bound $d(f)$, the canonical inclusion $i$ has bound 0 , and the canonical retraction $r$ is a homotopy equivalence of bound 0 with the standard homotopy inverse $j$. Since $i \simeq j f 1_{X}$ by a homotopy of bound 0 , if $\pi_{n}(f)$ (respectively $\left.H_{n}(\tilde{f})\right)$ is an $(a, b)$-isomorphism, then $\pi_{n}(i)$ (respectively $\left.H_{n}(\tilde{l})\right)$ is a $(2 a, 2 a+b)$-isomorphism by Lemma 1.2. Finally, if $i$ is a homotopy equivalence of bound $d$, then $f$ is a homotopy equivalence of bound $2(d+d(f))$ by Lemma 1.1 . This completes the reduction step.

\section{The proofs of the Bounded and Thin Whitehead Theorems}

The "only if" part of both Whitehead Theorems is left to the reader. The "if" part of the Thin Theorem is an immediate corollary of the Bounded Theorem. Indeed for any $\varepsilon>0$, one may choose $\delta>0$ with $L_{N, M}\left(0, d_{2}, d_{3}, d_{4}\right)<\varepsilon$ whenever $d_{i}<\delta(i=2,3,4)$. Then one only has to subdivide $X$ and $Y$ to get $d_{X}$ and $d_{Y}$ less than $\delta$.

By the results of $\S 1$, we need only prove the "if" part of the Bounded Whitehead Theorem for the case of an inclusion $i:(X, p) \rightarrow(Y, q)$ with $p=q i$.

The proof of the following lemma is an easy exercise.

Lemma 2.1. Let $(Y, X, q)$ be a pair of $C W$ complexes over $Z$. Then

(i) If $\pi_{k}(i)$ is a $(0, b)$-isomorphism for $k=n-1, n$ and some $n \geq 0$, then $\pi_{n}(Y, X, q) 2 b$-vanishes.

(ii) If $\pi_{k}(Y, X, q)$ b-vanishes for $k=n, n+1$ and some $n \geq 0$, then $\pi_{n}(i)$ is a $(0, b)$-isomorphism.

Let $i$ satisfy Condition (1) in the Bounded Whitehead Theorem. By Lemma 1.2 (i), we may assume $a=0$. Since $\pi_{n}(Y, X, q) 2 b$-vanishes for $n \geq-1$, the result follows from Corollary 1.5. Note that the assumptions concerning $\pi_{n}(i)$ for $i \leq 1$ are needed to conclude that $\pi_{n}(Y, X, q) 2 b$-vanishes for $n \leq 2$.

If $i$ satisfies Condition (2) in the Bounded Whitehead Theorem, we need the following weak form of a Bounded Hurewicz Theorem (cf. [Ch]) to finish the proof:

Proposition 2.2. For each integer $M \geq 0$, there is a linear function $L_{M}\left(d_{1}, d_{2}, d_{3}\right)$ for which the following holds: Let $(Y, X, q)$ be a bounded pair of $C W$ complexes over $Z$ with inclusion $i:(X, p) \rightarrow(Y, q)$ where $p=q \mid Y$, and let $a, b$ be real numbers such that

(i) $\pi_{n}(i)$ is an $(a, b)$-isomorphism for $n=-1,0,1$ and

(ii) $H_{n}(\tilde{l})$ is an $(a, b)$-isomorphism for all $n \geq 2$.

Then $\pi_{n}(i)$ is a $(0, L)$-isomorphism for all $n \geq-1$ for $L=L_{\operatorname{dim} Y}\left(a, b, d_{Y}\right)$. Proof. By Lemma 1.2(i), we may assume $a=0$. Then Proposition 2.2 is really a statement about the behavior of the functors $\pi_{n}(-, x)$ (with $x \in p^{-1} B$ ) and 
$H_{n}(-)$ applied to one of the diagrams

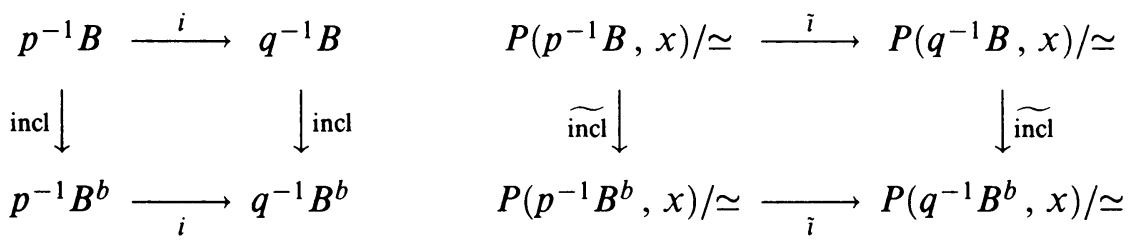

Let $Y_{B}$ be the smallest subcomplex of $Y$ containing $q^{-1} B$ (cf. [AM2, Example 1.6]) and notice that $q^{-1} B \subseteq Y_{B} \subseteq q^{-1} B^{\operatorname{dim} Y d_{Y}}$. These inclusions and the corresponding ones in $X$ allow us to concentrate instead on the results of applying $\pi_{n}(-, x)$ and $H_{n}(-)$ to diagrams of the form

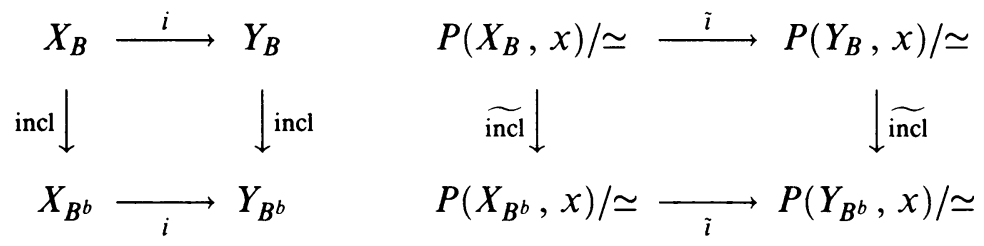

Thus we assume that $H_{n}(\tilde{l})$ is a $(0, b)$-isomorphism for $n \geq 2$, and we have to show that $\pi_{n}(i)$ is a $(0, L)$-isomorphism for $n \geq 2$.

We note that $\widetilde{Y}(x, B)=P\left(Y_{B}, x\right) / \simeq$ is the (honest) universal covering space of the component of $Y_{B}$ containing $x$. Let $\bar{l}: \bar{X}(x, B) \rightarrow \tilde{Y}(x, B)$ be the inclusion of the image of $\tilde{l}: \widetilde{X}(x, B) \rightarrow \widetilde{Y}(x, B)$ and $\eta: \widetilde{X}(x, B) \rightarrow \bar{X}(x, B)$ the corresponding quotient map. Since $\bar{X}(x, B)=P\left(X_{B}, x\right) / \sim$ where $\sim$ denotes homotopy in $Y_{B}$ relative to endpoints and since $\pi_{1}(i)$ is a $(0, b)$-isomorphism, there is a map $g: \bar{X}(x, B) \rightarrow \widetilde{X}\left(x, B^{b}\right)$ such that $g \eta: \widetilde{X}(x, B) \rightarrow \widetilde{X}\left(x, B^{b}\right)$ and $\eta g: \bar{X}(x, B) \rightarrow \bar{X}\left(x, B^{b}\right)$ are induced by the inclusion $B \subseteq B^{b}$. From this and the fact that each $\widetilde{X}(x, B)$ is simply connected, it is easily seen that for $d=2 b$

(i) $\pi_{1}(\bar{X}) d$-vanishes; and

(ii) $H_{n}(\bar{l})$ is a $(0, d)$-isomorphism for all $n \geq 2$.

Lemma 2.3. There is a linear function $L_{M}(d)$ so that $\pi_{n}(\bar{l})$ is a $\left(0, L_{M}(d)\right)$ isomorphism for all $n \geq 2$ and $M=\operatorname{dim} Y$.

Since the projections $(\bar{X}(x, B), \bar{x}) \rightarrow\left(X_{B}, x\right)$ and $(\tilde{Y}(x, B), \tilde{x}) \rightarrow\left(Y_{B}, x\right)$, induce isomorphisms on $\pi_{n}(-)$ (for $n \geq 2$ ) that are compatible with the homomorphisms induced by "inclusions" of the form $(x, B) \rightarrow\left(x, B^{d}\right)$, it follows from Lemma 2.3 that $\pi_{n}(i)$ is a $\left(0, L_{M}(d)\right)$-isomorphism for all $n \geq 2$. This completes the proof of Proposition 2.2.

Proof of Lemma 2.3. For notational simplicity, let $\tilde{Y}(x, B)=U(x, B)$, $\bar{X}(x, B)=V(x, B)$, and $\bar{l}=j$. Then $(U(x, B), V(x, B))$ is a pair of pointed $\mathrm{CW}$ complexes of dimension at most $M$. Because of (i), (ii) actually holds for all $n \geq 0$. Hence by the homology version of Lemma $2.1, H_{n}(U, V) 2 d$ vanishes for all $n \geq 0$ and by Lemma 2.1 , it suffices to show that $\pi_{n}(U, V)$ $L_{M}(d)$-vanishes for $n \geq 2$. Let $n \geq 2$, and assume inductively that for some linear function $L_{n}, \pi_{k}(U, V) L_{n}(d)$-vanishes for $0 \leq k<n$. An inspection of the homotopy exact sequence for $(U(x, B), V(x, B))$ shows that we may 
take $L_{2}(d)=0$. For the inductive step, it suffices to show that the Hurewicz map $\varphi: \pi_{n}(U, V) \rightarrow H_{n}(U, V)$ is a $\left(0, c_{n} d\right)$-isomorphism for a suitable constant $c_{n}$.

Our proof for this follows [S, pp. $387 \mathrm{ff}]$. We recall that for any pair of pointed spaces $(Y, B)$ and any integer $m \geq 0, \Delta(Y, B)^{(m)}$ is the subcomplex of the singular chain complex $\Delta(Y)$ generated by singular simplices $\sigma: \Delta^{q} \rightarrow Y$ that take all vertices to $*$ and the $m$-skeleton into $B$. Let $H_{*}^{(m)}(Y, B)=$ $H_{*}\left(\Delta(Y, B)^{(m)} / \Delta(Y, B)^{(m)} \cap \Delta(B)\right)$. A result of [S, p. 394] shows that for any $(x, B)$ with $p(x) \in B \subseteq Z$, the Hurewicz map $\varphi(x, B)$ fits into a commutative diagram

$$
\begin{array}{cc}
\pi_{n}(U(x, B), V(x, B)) \stackrel{\varphi(x, B)}{\longrightarrow} & H_{n}(U(x, B), V(x, B)) \\
\eta_{\pi}(x, B) \downarrow & \uparrow \eta_{H}(x, B) \\
\pi_{n}^{\prime}(U(x, B), V(x, B)) \underset{\varphi^{\prime \prime}(x, B)}{\longrightarrow} H_{n}^{(n-1)}(U(x, B), V(x, B))
\end{array}
$$

Since $\pi_{1}(V(x, B)) \rightarrow \pi_{1}\left(V\left(x, B^{d}\right)\right)$ vanishes, $\eta_{\pi}$ is a $(0, d)$-isomorphism. An argument of [S, p. 397] shows that $\varphi^{\prime \prime}(x, B)$ is an honest (i.e., a $(0,0)$-) isomorphism. By Lemma 1.2 , it now suffices to show that $\eta_{H}$ is a $\left(0, c_{n} d\right)$ isomorphism for a suitable constant $c_{n}$.

For this proof, we fix $(x, B)$ and write $U, V, U^{r}$, and $V^{r}$ for $U(x, B)$, $V(x, B), U\left(x, B^{r}\right)$, and $V\left(x, B^{r}\right)$, respectively. There is an exact sequence

$$
\cdots \rightarrow \mathscr{H}_{q+1}^{(n-1)}(U, V) \rightarrow H_{q}^{(n-1)}(U, V) \stackrel{\eta_{H}}{\longrightarrow} H_{q}(U, V) \rightarrow \mathscr{H}_{q}^{(n-1)}(U, V) \rightarrow \cdots
$$

where $\mathscr{H}_{*}^{(n-1)}(U, V)$ is the homology of $\Delta(U) / \Delta(U, V)^{(n-1)}$. By the homology analogue of Lemma 2.1 , it suffices to show that $\mathscr{H}_{*}^{(n-1)}(U, V) c_{n} d$ vanishes. Since there is a short exact sequence $\Delta(U, V)^{(n-1)} \rightarrow \Delta(U) \rightarrow$ $\Delta(U) / \Delta(U, V)^{(n-1)}$, this means that we must show that the inclusion $l: \Delta(U, V)^{(n-1)} \rightarrow \Delta(U)$ induces a $\left(0, c_{n} d\right)$-isomorphism on homology.

Let $\sigma: \Delta^{q} \rightarrow U$ be a singular simplex. Following [S, pp. 392-3], we claim that there is a map $P(\sigma): \Delta^{q} \times I \rightarrow U^{q d}$ such that

(i) $P(\sigma)(-, 0)=\sigma$;

(ii) $P(\sigma)(-, 1) \in \Delta\left(U^{q d}, V^{q d}\right)^{(n-1)}$;

(iii) if $\sigma \in \Delta(U, V)^{(n-1)}$, then $P(\sigma)(-, 1)=\sigma$; and

(iv) if $e^{i}: \Delta^{q-1} \rightarrow \Delta^{q}$ is the inclusion into the $i$ th face, then $P(\sigma)\left(e^{i} \times 1\right)=$ $P\left(\sigma e^{i}\right)$.

The construction of $P(\sigma)$ is by induction on $q$. For $q=0, P(\sigma)$ is simply a path in $U$ from the point $\sigma\left(\Delta^{0}\right)$ to the basepoint in $V$, so the induction can be started.

Next consider the inductive step from $q-1$ to $q$ when $1 \leq q \leq n-1$. If $\sigma \in \Delta_{q}(U, V)^{(n-1)}$, let $P(\sigma)=\sigma \pi$ where $\pi: \Delta^{q} \times I \rightarrow \Delta^{q}$ is the projection. In the general case, (i) and (iv) serve to define $P(\sigma) \mid\left(\Delta^{q} \times 0 \cup \dot{\Delta}^{q} \times I\right)$ as a map into $U^{(q-1) d}$. Since $P(\sigma) \mid\left(\Delta^{q} \times 0 \cup \dot{\Delta}^{q} \times I\right)$ maps $\dot{\Delta}^{q} \times 1$ into $V^{(q-1) d}$, it represents an element $\alpha \in \pi_{q}\left(U^{(q-1) d}, V^{(q-1) d}\right)$. Since $\pi_{q}(U, V) d$-vanishes, $j_{*}(\alpha)=0$ in $\pi_{q}\left(U^{q d}, V^{q d}\right)$. An extension with the desired properties can now be seen to exist. 
Finally, consider the inductive step from $q-1$ to $q$ when $q>n-1$. As before, let $P(\sigma)=\sigma \pi$, if $\sigma \in \Delta_{q}(U, V)^{(n-1)}$. If $\sigma \notin \Delta_{q}(U, V)^{(n-1)}$, then any extension of $P(\sigma) \mid\left(\Delta^{q} \times 0 \cup \dot{\Delta}^{q} \times I\right)$ can be used. Note that an extension into $U^{(n-1) d}$ exists since $\Delta^{q} \times I$ retracts to $\left(\Delta^{q} \times 0 \cup \dot{\Delta}^{q} \times I\right)$.

The function $\sigma \rightarrow P(\sigma)(-, 1)$ defines a chain map $\rho$ such that $\rho: \Delta(U) \rightarrow$ $\Delta\left(U^{(n-1) d}, V^{(n-1) d}\right)^{(n-1)}$ by (iv); while (ii) shows that incl ${ }_{*}=\rho l: \Delta(U, V)^{(n-1)}$ $\rightarrow \Delta\left(U^{(n-1) d}, V^{(n-1) d}\right)^{(n-1)}$. In addition, the maps $P(\sigma)$ can be used to construct a chain homotopy incl $_{*} \simeq \imath \rho: \Delta(U) \rightarrow \Delta\left(U^{(n-1) d}\right)$. It is now easy to finish the proof.

\section{A special case of the Bounded Whitehead Theorem}

Let $(X, p)$ be a bounded CW complex. We say $\pi_{n}(X)$ is uniformly locally presented of bound $d$ (cf. [AM1, p. 261] or [Pe]) if for every ball $B \subseteq Z$,

(i) $p^{-1} B^{d} \neq \varnothing$ and

(ii) for every $x \in p^{-1} B, i_{1 *}$ is onto and $\operatorname{ker} i_{0 *} \subseteq \operatorname{ker} j_{*}$ in the following diagram where $i_{0}, i_{1}$, and $j$ denote inclusions:

$$
\begin{array}{cc}
\pi_{n}\left(p^{-1} B, x\right) \stackrel{i_{0 *}}{\longrightarrow} \pi_{n}(X, x) \\
j_{*} & \downarrow 1 \\
\pi_{n}\left(p^{-1} B^{d}, x\right) \stackrel{i_{*}}{\longrightarrow} \pi_{n}(X, x)
\end{array}
$$

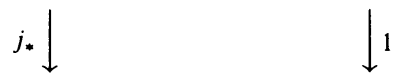

Let $(X, p)$ be a bounded CW complex over $Z$ and $\rho: \tilde{X} \rightarrow X$ be the universal cover of $X$. Then $(\tilde{X}, p \rho)$ is also a bounded CW complex over $Z$. In general, the spaces $\tilde{X}(x, B)$, etc. in $(0.2)$ cannot be replaced by the spaces $(p \rho)^{-1} B$, etc. If $\pi_{n}(X)$ is uniformly locally presented for $n=0,1$, then such a substitution can be made and the following theorem is then a special case of the Bounded Whitehead Theorem:

Theorem 3.1. Let $f:(X, p) \rightarrow(Y, q)$ be a map of bound a between bounded $C W$ complexes over a metric space $Z$. Suppose that $\pi_{n}(X)$ and $\pi_{n}(Y) \quad(n=$ $0,1)$ are uniformly locally presented of bound $b$. Then $f$ is a bounded homotopy equivalence if and only if

(1) for any $x \in X, f_{*}: \pi_{n}(X, x) \rightarrow \pi_{n}(Y, f(x))(n=0,1)$ is an isomorphism; and

(2) for some $c \geq b$ and for every $n \geq 2$, every ball $B \subseteq Z$, and every $x \in p^{-1} B$, we have $\operatorname{im} \tilde{\jmath}_{1 *} \subseteq \operatorname{im} \tilde{f}_{1 *}$ and $\operatorname{ker} \tilde{f}_{0 *} \subseteq \operatorname{ker} \tilde{j}_{0 *}$ in the diagram

$$
\begin{array}{cc}
H_{n}\left((p \rho)^{-1} B\right) \stackrel{\tilde{f}_{0 *}}{\longrightarrow} & H_{n}\left((q \rho)^{-1} B^{a}\right) \\
\tilde{j}_{0 *} \downarrow & \downarrow^{j_{1 *}} \\
H_{n}\left((p \rho)^{-1} B^{c}\right) \stackrel{\tilde{f}_{1 *}}{\longrightarrow} & H_{n}\left((q \rho)^{-1} B^{a+c}\right)
\end{array}
$$

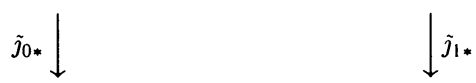

Here $\widetilde{W} \stackrel{\rho}{\longrightarrow} W$ is the universal cover of $W(W=X, Y)$ and $\tilde{f}:(\tilde{X}, p \rho) \rightarrow$ $(\tilde{Y}, q \rho)$ is a lift of $f$ to universal covers. 
The reader may augment this theorem with a bound $L_{N, M}\left(a, c, d_{X}, d_{Y}\right)$ for the homotopy equivalence obtained and formulate and prove the resulting "thin" version.

\section{ACKNOWLEDGMENT}

This paper arose from trying to understand some of the referee's comments on the original draft of [AM2]. We thank the referee of that paper for suggesting that the ideas in [AM2] might be adaptable to the present setting. The first named author would also like to thank the Mathematics Department of the University of Notre Dame for its hospitality during the period when this paper was written.

\section{REFERENCES}

[AM1] D. R. Anderson and H. J. Munkholm, Boundedly controlled topology, Lecture Notes in Math., vol. 1323, Springer-Verlag, New York and Heidelberg, 1988.

[AM2] _ The boundedly controlled Whitehead Theorem, Proc. Amer. Math. Soc. 117 (1992), 561-568.

[Ch] T. A. Chapman, Controlled boundary and h-cobordism theorems, Trans. Amer. Math. Soc. 280 (1983), pp. 73-95.

[Pe] E. K. Pedersen, On the bounded and thin h-cobordism theorem parametrized over $\mathbb{R}^{k}$, Transformation Groups (Poznan 1985), Lecture Notes in Math., vol. 1217, Springer-Verlag, New York and Heidelberg, 1986, pp. 306-319.

[S] E. H. Spanier, Algebraic topology, McGraw-Hill, New York, 1966.

Department of Mathematics, Syracuse University, Syracuse, New York 13244

Institut for Matematik og Datalogi, Odense Universitet, DK 5230 Odense M, DenMark 